\title{
Microtome: histopathology day book based on a microcomputer system
}

\author{
D ROBERTS-JONES, J McCLURE \\ From the Department of Histopathology, University Hospital of South Manchester, Manchester
}

SUMMARY The development of a histopathology day book based on a microcomputer is described. The system has the capacity to search on file records for details of previous specimens from current patients. It also possesses a SNOP/SNOMED input and search system that can aggregate data for analytical and other purposes. The system is relatively inexpensive and is user friendly. It has been developed within and is exclusively devoted to the requirements of a histopathology department and is designed to permit expansion to a multiuser system.

The acquisition, storage, and retrieval of information are important functions of any pathology laboratory. In haematology, biochemistry, and (to a lesser extent) microbiology laboratories results are largely in the form of numerical data, and for several years computers have been used to accumulate, store, and manipulate these data. In histopathology computers have also been used but to a much lesser extent than in the related disciplines. Part of the reason for this is the fact that histopathological reports are written in complex language. Although this can be summarised by an encodement process using the SNOP/SNOMED systems, automatic encodement facilities ${ }^{1}$ are not generally available and, when they are available, looking up the appropriate manual is prone to error and can be tedious.

The computers used in histopathology laboratories have generally been mainframe or mini computers and have been geared towards generating reports (word processing) and compiling statistical information. Such systems are expensive and require the services of professional programmers. Often they are shared with other laboratories or hospital records systems, or there is a connection to a regional computer by an expensive telephone link. ${ }^{2-8}$

In 1983 we undertook a critical examination of the information storage and retrieval systems then in use in the histopathology department of this hospital. The systems were entirely manual and based essentially on a traditional day book comprising a ledger and card index. The department provides a comprehensive diagnostic service (including non-

Accepted for publication 22 May 1985 gynaecological cytopathology) to a busy major teaching hospital and receives some 12000 specimens (excluding necropsy material) every year. Therefore, although initially satisfactory, in time the archive became oversized and somewhat cumbersome, especially the component that dealt with datz retrieval. It was particularly difficult to determine whether previous biopsy material had been receive $f$ from the patients whose current specimens were being handled. The advent of endoscopic biopsy techniques has resulted in many specimens being obtained from patients over time, and archival material is constantly needed to confirm diagnosis and assess the efficacy of treatment.

We did not have access to mainframe or mini computers or the capital to devise a system capable of using these, but we were interested in microcomputers and our examination of the existing laboratory system was to determine if a microcomputer system could be developed that would improve the efficiency of part or all of the existing procedures. This report concerns our analysis of the historical system and the development of a system based on microcomputers, with particular reference to design philosphy, hardware, data retrieval, and techniques of analysis.

\section{THE HISTORICAL SYSTEM}

Fig. 1 shows how the historical system worked. A specimen accompanied by a request card was received in the laboratory and given a laboratory accession number, comprising two digits indicating the year and the maximum number of specimens received in a given year. The patient's personal details, specimen number, clinical consultant, and 


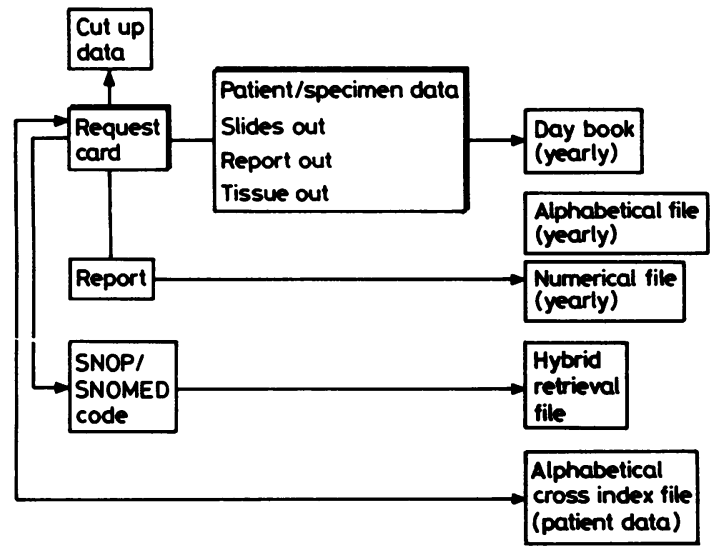

Fig. 1 Flow chart illustrating historical data recording and retrieval system.

the histopathologist were then recorded in a ledger. Data such as the date of issue of the report and disposal of preserved wet tissue were recorded later. The description of the gross specimen made at cut up was recorded on the request card, which was stored in an alphabetical file. Data from the request card were transferred to the final report issued to the responsible clinical consultant and a copy kept in a numerical file (based on accession number) in the laboratory. The day book and alphabetical and numerical files were accumulated on a yearly basis.

In addition, the responsible pathologist encoded the diagnosis using the SNOP and, latterly, the SNOMED systems. The accession number was then recorded in a card index file under the headings of (T)opography and (M)orphology (hybrid retrieval file). Another alphabetical cross index file was also maintained, which was based on the initial of the patient's surname but included forename, medical records file number, and accession numbers of all material from that patient. This file was designed to permit retrieval of details of both previous and current biopsy reports from the same patient. The laboratory had one clerical officer who gave accession numbers to the specimens and searched the alphabetical cross index file for previous accession numbers, a process that was cumbersome and time consuming, particularly when the work was performed by less skilled and experienced staff.

\section{DESIGN PHILOSOPHY}

It was evident from our analysis that the information in the day book could form a potential database, and we thought that a microcomputer system could be devised relatively easily to perform this task. What advantages, if any, would an electronic day book have? We needed a system that would allow a search of the database to determine the details of previous material from current cases and with the capacity to perform analyses and generate statistics. Although a microcomputer had been used as a word processor to generate histopathology reports, ${ }^{9}$ we decided not to pursue this further for the time being.

\section{Material and methods}

The hardware used in establishing the microcomputer system comprised the following: Apple II Europlus $48 \mathrm{~K}$ microcomputer; floppy disk drive and controller card; Epson MX80 FT/III printer; Grappler $64 \mathrm{~K}$ printer buffer card; Z80 second processor card $(2 \mathrm{MHz}) ; 80$ column card; amber phosphor high resolution monitor; Microsoft 16K RAM card; and a Corvus Winchester disk drive (5Mb).

The microcomputer, printer, and cards were supplied by Fairhurst Instruments, Wilmslow, Cheshire, and the disc drive was loaned by Dr S Lucas, Faculty of Medicine Computational Group, University of Manchester.

The software used to develop the system comprised: Microsoft basic interpreter (MBASIC); Microsoft basic compiler (BASCOM); and a Keele codes CLIP (Winchester Backup Utility). Programmes operate under CPM version (2.2).

\section{Results}

Each morning the system is activated and the operator is requested to insert a password, the date, and the initials of the responsible histopathologists. Input of new data can then be started. On receipt of a new specimen and its accompanying request card the laboratory accession number is ascribed and the data are recorded in the microcomputer system. Essentially, these are the patient's personal details and some details of the specimen. After macroscopic examination, cutting up of the specimen, and the processing of tissue blocks details of the number of blocks taken, the number of slides issued to the histopathologists, and the issue of the report are recorded. The system automatically dates these events.

During input of a new record the operator is prompted to complete each data field in a preset order. The operator is then given the chance of editing any field before committing the data to file. When extending an existing record the operator selects the field that needs amending (Figs 2-4). For the surname the system accepts letters $A$ to $Z$ and a hyphen only (the field is truncated to a maximum of 14 characters). For the forename it again accepts $A$ to $\mathrm{Z}$ but is set to seven characters. If numerical data are added to this field then the program assumes 

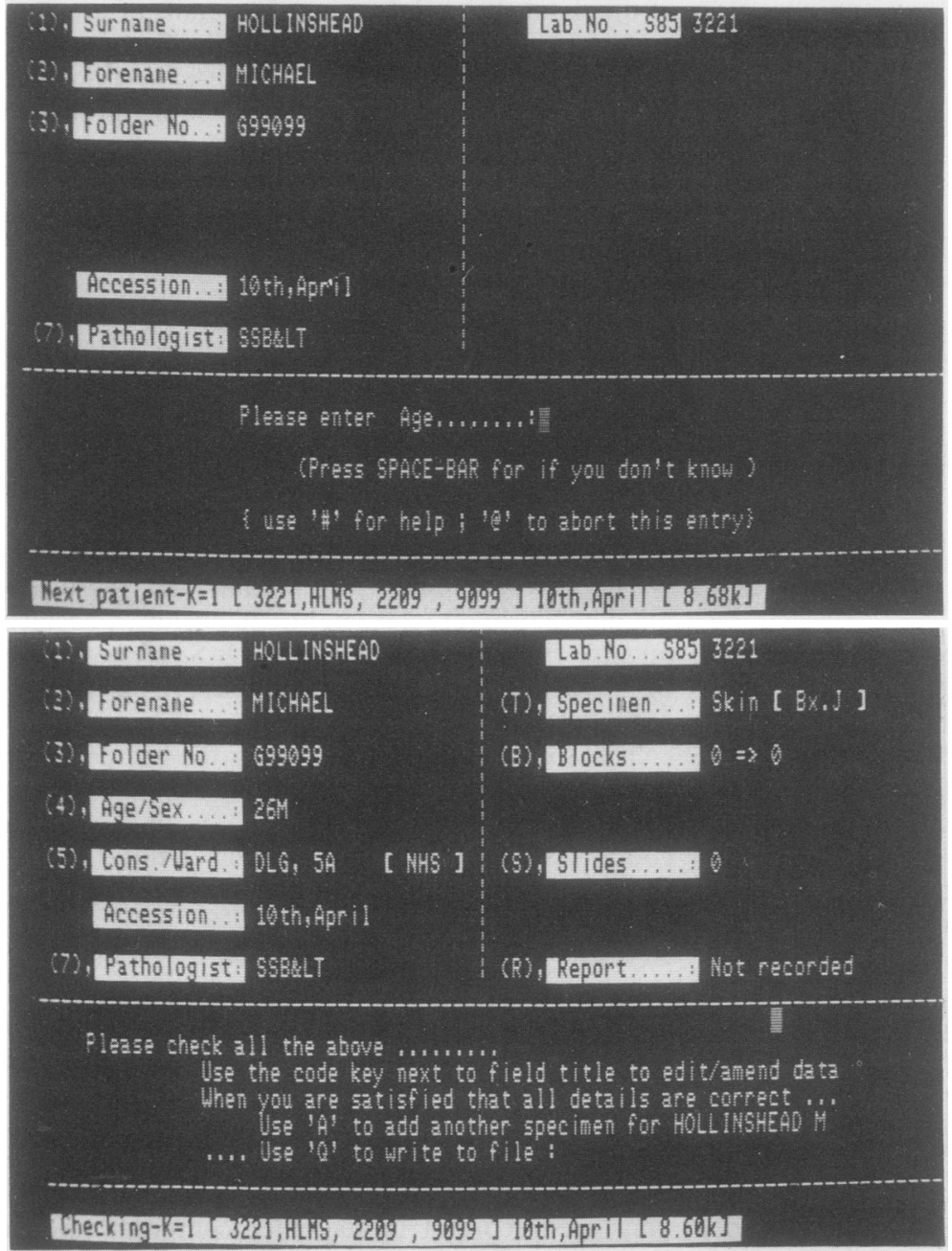

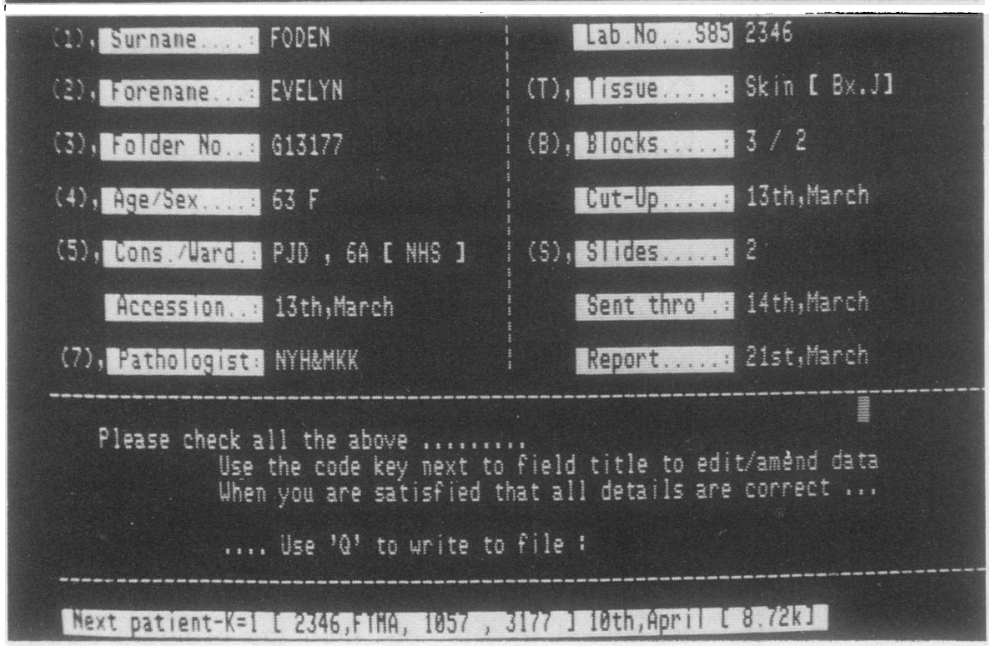

Fig. 2 Laboratory number, accession date, and initials of responsible pathologist(s) are automatically supplied by the system when the patient's personal data are entered.

Fig. 3 Data input is now ational specimens for this patient may now be added. All data. to left of vertical line will be remembered by the system.

Fig. 4 Complete record of fictitious patient. Dates are automatically ascribed. 
that the specimen is from a laboratory animal and the subsequent program flow is modified. The patient's medical record number is accepted in any printable characters to a maximum of seven; the age is accepted as numerical data in the range 0 to 120 . If a zero is entered the program requests the date of birth. Gender is entered as $M$ or $F$ or $O$ (unknown). The ward or department in which the patient is being treated is indicated by any printable characters to a maximum of four. If material is sent from outside the hospital standard prefixes are used to indicate the sources. Thus ${ }^{*}$ indicates another NHS hospital: $\$$ a private hospital; ! a general practice or health centre; / the university or Public Health Laboratory Service.

The initials of the referring clinical consultant are accepted as $A$ to $Z$ with a maximum length of four characters. Data about the specimen are given in a single alphanumerical code: $T=$ large specimen; $H$ $=$ medium sized specimen $; \mathrm{B}=$ small specimen $; \mathrm{F}=$ frozen section; $E=$ specimen submitted for transmission electron microscopy; and $\mathrm{C}=$ specimen for cytological examination. The system automatically decodes these to the visual display unit (VDU). The anatomical site of origin of the specimen is indicated by a modified SNOP topography code of two digits, and the system again automatically decodes to the VDU screen. The responsible histopathologist(s) and the accession date are entered automatically, having been recorded during the morning $\log$ in procedure. Additional fields are added in the edit mode only. The number of tissue blocks is recorded as a numerical input $(<99)$ with the cut up date being logged automatically. Again the number of slides issued to the pathologist is recorded as a numerical input (<999) with automatic dating. The issue of the report is recorded by a single keystroke (R) with automatic logging of the date.

After the insertion of these data a residue of 60 bytes (alphanumerical characters) for the encodement of the diagnosis using the SNOP/SNOMED systems is available. These are usually added after the issue of the report as the encodement is done by the histopathologists. The prime reference key is the laboratory accession number, which displays all the above data except the SNOP/SNOMED coding and allows editing of any item. Each completed record occupies 128 bytes (Table 1.) A single byte in each record has been reserved for record locking should funds become available in the future to develop a multioperator system.

The database system may be defined as a "finite state automaton." This means that at any time during operation it is aware of factors such as the highest laboratory accession number used to date, whether new data are being added, and whether
Table 1 Structure of completed record indicated by number of bytes ascribed to each component (total number of bytes $=128$ )

\begin{tabular}{lr}
\hline Record lock & 1 \\
Accession date & 2 \\
Surname & 14 \\
Forename & 7 \\
Medical record number & 7 \\
Age/gender & 2 \\
Referring clinician & 4 \\
Ward or departmental source & 4 \\
Status (NHS/private) & 1 \\
Responsible histopathologist(s) & 6 \\
Indication of specimen size and anatomical site of origin & 2 \\
Cut up data (pieces and blocks) & 2 \\
Cut up date & 2 \\
Number of slides prepared & 2 \\
Date slides sent to histopathologist & 2 \\
Date case reported & 2 \\
& 2 \\
Surname $\quad$ forward & 2 \\
Pointers \{ Folder number forward & 2 \\
$\quad$ Folder number backward & 60 \\
SNOP/SNOMED code & \\
\hline
\end{tabular}

existing details are being modified. The program is capable of modifying its action in response to the input. The data are stored as indexed sequential files in laboratory accession number order with each year's data filed separately. At the moment the maximum number of specimens that can be handled annually is 32767 . The maximum number of records depends on the size of the storage medium (currently five megabytes, equivalent to two and a half years of data plus programs). More storage can be added to the system, although we would not expect to need more than 10 years of data on line.

At the end of each working day a daily acquisition list is printed. This is stored as hard copy. It lists all specimens received that day, together with details of previous material from each patient, the responsible histopathologist, and source of specimen.

It is a relatively simple matter to create a facility for matching current names and medical record numbers with filed data, but successful retrieval depends on the current request cards being accurately completed with respect to these details. In practice data completed on pathology request cards are notoriously inaccurate. There may be simple misspelling of a surname-BATES instead of BATTS - or there may be homonymic errors such as FITZMORRIS instead of FITZMAURICE, or the surname and forename may be inverted. This is a particular problem with unfamiliar foreign names. A woman may not have her maiden name changed in her medical record after marriage. Another common event is the issue of two separate medical record numbers to one patient. Forenames may be omitted, misspelled, or given as just an initial. Problems may also arise with the medical record number: 
at this hospital this is composed of a letter (currently G) followed by a number that has leading zeros. The number may be completely omitted or presented in a non-standard way-for example, G00138 may be presented as $\mathrm{G} 138$ or even $\mathrm{G} / 138$.

Given the number of possible errors, a system that depends on an exact match will have a lowered positive retrieval rate. The database was therefore designed to catch most of these errors using the concept of incomplete or "fuzzy" matching. The operation of fuzzy matching is totally transparent to the operator. Names are reduced to a number between 1 and 10000 . Vowels are ignored (except where they are the initial letter), and the same numerical value is ascribed to each similarly sounding consonant group. A similar transformation is performed on the medical record number removing both prefixes and suffixes, so that G00123 $=123 / 85$. This technique is based on the well known SOUNDEX algorithm. ${ }^{10}$

Each record contains four pointers to other records deemed to be similar by having identical fuzzy attributes. The pointers link records both retrospectively and prospectively, which is essential for automatic repair of the database after a change of either name or medical record number. ${ }^{11}$

Fig. 5 illustrates a fictitious segment of a daily print out. There are 11 specimen entries. In the first item 85.02481 is the laboratory accession number, GUBBINS the surname, Annie the forename, E92988 the medical record number, 5B the hospital ward (the (-) suffix here indicates NHS), SSB the responsible histopathologist, and Breast the anatomical site of the specimen. Also given in the first item are details of previous specimens from this patient. An exact match has been obtained with the surname and medical record number. Note, however, the variation of forename. Also included are the SNOP/ SNOMED encodements of previous diagnoses. Item number four refers to SILLY, Sally and item nine to DAFT, Sally. An exact match is given on the basis of the medical record number.

A fuzzy match is illustrated by items five and 11 . In the first the names are "BUMBY, Joan" and the number is $A 49748$, while in the second they are "BAMBI, Joan" and A49746. The first is printed with the second as a fuzzy match. In the print out such a match is flagged as ??\#. SNOP/SNOMED codes are not printed for fuzzy matches. On noting the exact and fuzzy matches the clerical officer will retrieve the appropriate previous reports, which are then sent to the responsible histopathologist with the current slides. The histopathologist then decides if the fuzzy matches are true and if the previous slides are required for review in the light of the current material.
The system also contains a SNOP/SNOMED input and search system. Input into this may be by a valid SNOP code; a valid SNOMED code; a SNOMED mnemonic (for example, HO = history of); and segments of free text. If the first three of these items are on file the system will automatically decode input to the screen. If the keyed items are not on file the program will request that it be taught the appropriate translation. If a specific disease process is characterised by these encodements and keyed into the system then a print out of the details of all the patients with this diagnostic encodement can be generated. If more than 50 such records exist then a histogram of numbers against five year intervals of age is drawn for each gender. For example, the database containing 14994 records was searched for examples of prostatic hyperplasia (T77M730) and fibroadenoma of the breast (T04M9010). Of the records searched, 9433 contained codes $(62.91 \%)$. A positive match for T77M730 was found in 219 of these, and Fig. 6 is the resulting histogram of frequency against age for those records in which the age was specified. Positive matches for T04M9010 were found in 109 records. Of these, 27 did not specify age. The histogram for the remainder is illustrated in Fig. 7.

\section{Discussion}

We have shown that a system based on a microcom puter can be developed to provide an electronic day book with a capacity to store, search, and retrieve data on previous specimens and to perform compilations for analytical, administrative, planning, and costing purposes. The precise details of these search, retrieval, and compilational facilities will be given elsewhere. A high degree of sophistication was achieved with a relatively modest system. We managed to borrow the main items of the system, but the total cost would be about $£ 4000$, a comparatively small sum when compared with the cost of mainframe computers and professionally written software packages. This is computational development on a shoestring, but our relative poverty was advantageous in that it forced us to think critically and carefully about existing and developed procedures and their appropriate form and function.

Foulis et al ${ }^{12}$ and Talamo and Losos ${ }^{13}$ have also reported a similar system for anatomical pathology. These systems are much larger than ours and are able to perform automated SNOP/SNOMED coding. Our system has been developed without the assistance of professional programmers. The system is user friendly and will cope with erroneous entries. It is designed to be used by clerical, secretarial, and laboratory staff with no formal training in computer 
Microtome: histopathology day book based on a microcomputer system

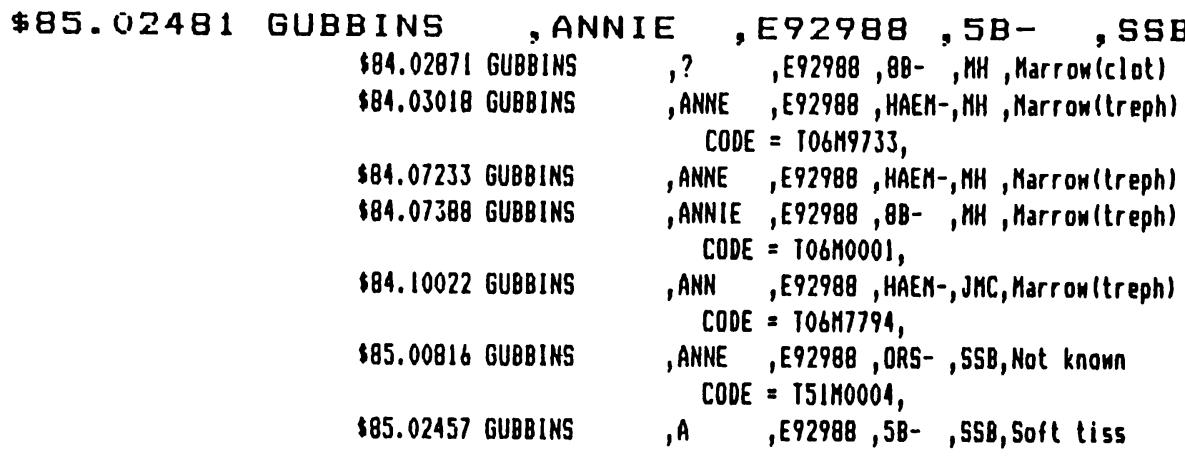

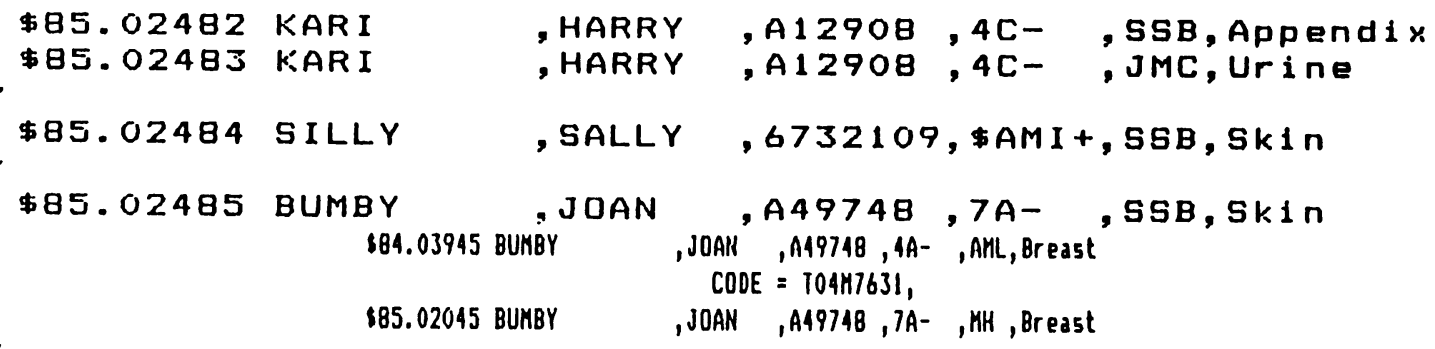

Fig. 5 Fictitious segment of daily print out. 


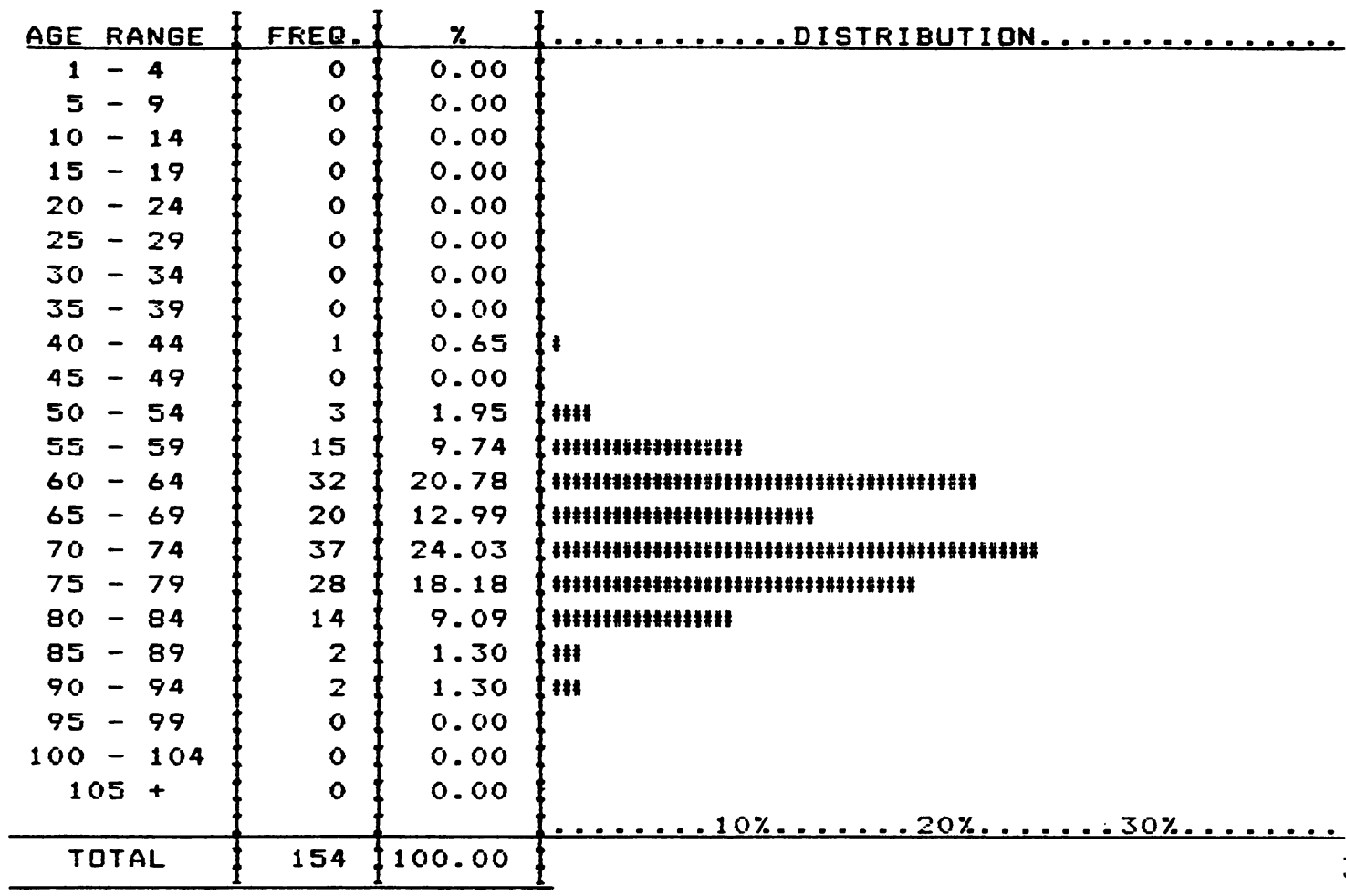

65 record(s) did not specify age.

Fig. 6 Occurrence of prostatic hyperplasia against age generated by a SNOP/SNOMED search of database.

operation. The system, self contained and wholly dedicated to histopathology, is fully under departmental control and is independent of other departmental hospital computer systems.

Particular features of our system include the retrieval process based on the operation of fuzzy matching and the capacity to generate lists and histogram displays of particular diagnoses, and all new submissions on an exact and fuzzy match basis for archival material. The system does not replace our clerical officer but allows her to concentrate on other much needed activities in the department.

Histopathologists, when confronted with unusual, classical, or difficult cases, often remember having seen similar cases in the past. With the new system they can obtain exact details about these previous cases in a short time. There is also an increased awareness of the principle of computing that "rubbish in" gets "rubbish out," and consequently there is an increased awareness of the need for accuracy in keying in the SNOP/SNOMED codes.

The development and success of the system required a considerable investment of effort, awareness of microcomputers, and exact (albeit amateur) computer literacy. If these coexist then microcomputers can be exploited for inhouse needs as opposed to adapting existing mainframe systems. Both approaches have their advocates, and it is not possible to predict which will become dominant. We see the development of systems based on microcomputers as a means of providing a cost effective viable alternative to much larger and more expensive mainframe computer systems for use in the histopathology department.

We thank Dr S Lucas and Mr J Fairhurst for support in the development of the system, Mrs C Hodgkinson and Mrs C Brown for pioneering its use, Mrs A Mellor for typing the manuscript, and $\mathrm{Mr} \mathrm{M}$ Hollinshead for the photographs. 


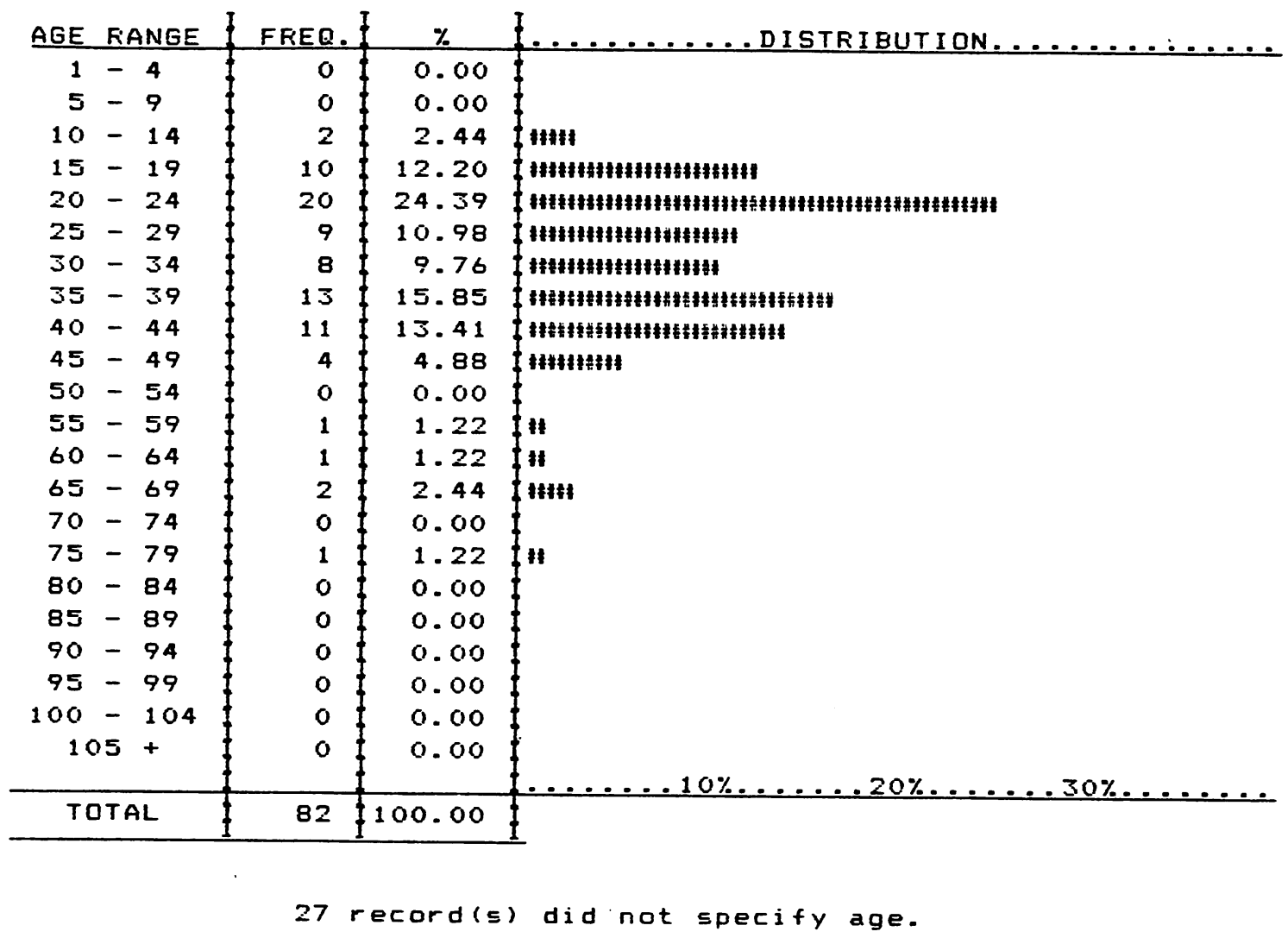

Fig. 7 Frequency of fibroadenoma of female breast generated by SNOP/SNOMED search of database.

\section{References}

' Coles EC, Slavin G. An evaluation of automatic coding of surgical pathology reports. J Clin Pathol 1976; 29:621-5.

${ }^{2}$ Codling BW, Macartney JC, Curran RC. An on-line computer system for histopathology reporting. J Clin Pathol 1977;30:1119-23.

${ }^{3}$ Robboy SJ, Altshuler BS, Chen HY. Retrieval in a computer assisted pathology encoding and reporting system (CAPER). Am J Clin Pathol 1981;75:654-61.

4 Foster EA, Stein A, Liberman D, Cooper C, Wolfe HJ. A computer-assisted surgical pathology system. Am J Clin Pathol 1982; 78:328-36.

swettenham KV, Nickols C, Berry CL. Computer programs in histopathology record keeping. J Clin Pathol 1982;35:40-4.

- Gallo P, DeBlasi Virginia. A computerized data bank of surgical pathology and cytopathology diagnoses. Virchows Arch (Pathol Anat) 1983;401:345-54.

' Swettenham KV, Nickols CD, Berry CL. Computer programs in cytology reporting and record keeping. J Clin Pathol 1983;36:856-9. subbuswamy SG, McCormick A, Peters EE. Computerization of histopathology/cytology records-use of a commercial data storage system. J Clin Pathol 1984;37:157-62.

' MacDonald RA, Pechet Giselle S, Lovenbury P. Surgical pathology reports with a portable microcomputer. Arch Pathol Lab Med 1982;106:666-9.

${ }^{10}$ Clarke D. Sounds familiar. Practical Computing 1984;7:90-2.

"Tremblay JP, Sorenson PG. An introduction to data structures with applications. 2nd ed. New York: McGraw-Hill, 1984.

${ }^{12}$ Foulis PR, Norbut AM, Mendelow H, Kessler GF. Pathology accessioning and retrieval system with encoding by computer PARSEC). A microcomputer-based system for anatomic pathology featuring automated SNOP coding and multiple administrative function. Am J Clin Pathol 1980;73:748-53.

${ }^{13}$ Talamo TS, Losos FJ. Surgical pathology accessioning and management on a multiuser hard disk microcomputer system. Arch Pathol Lab Med 1985; 109:19-23.

Requests for reprints to: Dr J McClure, Department of Histopathology, University Hospital of South Manchester, Nell Lane, Withington, Manchester M20 8LR, England. 